\title{
Electronic motion in a time-dependent two-centre model potential
}

\author{
D H Jakubassa-Amundsen $\dagger$ and J Macek $\ddagger$ \\ $\uparrow$ Physics Section, University of Munich, 8046 Garching, Federal Republic of Germany \\ $\ddagger$ Nielsen Physics Building, University of Tennessee, Knoxville, TN 37996-1200, USA
}

Received 20 December 1988, in final form 15 May 1989

\begin{abstract}
We calculate the propagator for an electron in a time-dependent quadratic potential which results from an expansion of the two-centre Coulomb field around its saddle point. Exact solutions are obtained for two types of time dependencies, corresponding to a straight line as well as a Coulomb trajectory for the internuclear motion.
\end{abstract}

\section{Introduction}

The final state of electrons ejected in energetic ion-atom collisions is, in general, dominated by one of the ionic potentials, either the target field in the case of low-energy or binary-peak electrons, or the projectile field for electrons with small momenta relative to the projectile (cusp electrons). Special interest has been devoted to situations where both fields influence the behaviour of the electrons. In the case of high-energy electrons ejected by projectile ions much heavier than the target, there is clear experimental evidence for two-centre effects in the electron spectra (Stolterfoht et al 1987). Alternatively, two-centre effects can be probed by considering electrons which propagate on the saddle of the potential surface produced by the projectile and the target ion in a near-symmetric collision (Meckbach et al 1986). Although apparently strong experimental evidence for the saddle-point electrons appearing as a ridge in the beam direction could not be reproduced (Suárez et al 1988), a peak structure for electron velocities around half the beam velocity has been observed at emission angles around $20^{\circ}$, both experimentally and in a Monte Carlo calculation (Olson et al 1987) and is ascribed to the presence of saddle-point electrons. A detailed theoretical study of these electrons requires, however, an explicit knowledge of their proper states.

An approach to formulating the appropriate wavefunction of the saddle-point electrons is considered in the present work. An, albeit approximate, wavefunction may be constructed by expanding the two-centre Coulomb potential about its saddle point. In this manner, an oscillator-type Schrödinger equation with time-dependent frequency is recovered, the treatment following closely the Wannier theory for threebody Coulomb break-up (Peterkop 1971, Feagin 1984). The wavefunction is most readily calculated from the corresponding propagator.

The propagator for a one-dimensional time-dependent attractive oscillator potential has already been calculated, either directly with the help of Feynman's path integral method (Khandekar and Lawande 1975), or by first simplifying the problem via a transformation of the timescale and so establishing, with the path integral method, a 
relation (Lawande and Dhara 1983) to the well known propagator for an oscillator with constant frequency (Feynman and Hibbs 1965). Later, an extension was made to oscillators with additional time dependence in the mass parameter and, for the calculation of the propagator, a spacetime transformation of the classical equations of motion was made in order to reduce the action integral to the one for a constant mass-constant frequency oscillator, thus avoiding the calculation of path integrals (Nassar et al 1986). Most recently, the three-dimensional case with inclusion of a time-varying magnetic field (with fixed orientation) has been solved employing the same methods (Nassar and Berg 1986). With an appropriate gauge, this magnetic field leads to a term in the Schrödinger equation proportional to the angular momentum operator and thus may conveniently be eliminated by transforming into a rotating coordinate system, provided the remaining Hamiltonian is rotationally invariant.

In all these approaches, the propagator is expressed analytically in terms of an unknown function which is a solution to an auxiliary differential equation containing the explicit time dependencies of the parameters. In this work, we show that the propagator can alternatively be obtained by calculating the complete set of quantum mechanical eigenfunctions of the appropriately transformed equations, thus avoiding the classical methods $(\$ 2)$. In this manner, the propagator for the time-dependent repulsive oscillator is found, which until now has only been achieved for the timeindependent case (Barton 1986). Subsequently, the time dependence of the propagator is calculated explicitly by solving the auxiliary differential equations for a special class of time-dependent frequencies $(\$ 3)$. Finally, the wavefunction is calculated for initial conditions corresponding to a plane wave and a Gaussian wavepacket, respectively (§4). Concluding remarks are given in $\S 5$. Atomic units $(\hbar=m=e=1)$ are used throughout.

\section{Formulation and evaluation of the propagator}

Let us assume a symmetric one-electron collision system with the nuclear charge of projectile and target denoted by $Q$. Choosing a reference frame centred at the midpoint between the two nuclei a distance $R(t)$ apart, and making an expansion of the potential around $\boldsymbol{r}=0$ where $\boldsymbol{r}$ is the electronic coordinate, one obtains the saddle-point potential

$$
\begin{gathered}
V(\boldsymbol{r})=-\frac{Q}{\left[x^{2}+y^{2}+(z-R / 2)^{2}\right]^{1 / 2}}-\frac{Q}{\left[x^{2}+y^{2}+(z+R / 2)^{2}\right]^{1 / 2}} \\
\simeq-\frac{4 Q}{R}\left(1-\frac{2 x^{2}}{R^{2}}-\frac{2 y^{2}}{R^{2}}+\frac{4 z^{2}}{R^{2}}\right) \quad r \ll R / 2 .
\end{gathered}
$$

The $z$ axis has been taken along the internuclear line. For non-head-on collisions the internuclear coordinate $\boldsymbol{R}(t)$ performs a rotation (in the $x z$ plane) which leads to an additional Coriolis coupling. Thus the approximate Schrödinger equation is

$\mathrm{i} \frac{\partial}{\partial t} \psi(\boldsymbol{r}, t)=\left(-\frac{\nabla^{2}}{2}-\frac{4 Q}{R}+\frac{8 Q}{R^{3}}\left(x^{2}+y^{2}\right)-\frac{16 Q}{R^{3}} z^{2}\right) \psi(\boldsymbol{r}, t)+\dot{\theta} \hat{L_{y}} \psi(\boldsymbol{r}, t)$

with $\dot{\theta}=b v / R^{2}, b$ the impact parameter, $v$ the collision velocity and $\hat{L}_{y}$ the angular momentum operator. As the Coriolis term implies a considerable complication it is usually neglected in the theory for Coulomb break-up. In the present case it may also be neglected for small collision velocities. In order to give an estimate for the upper 
limit on $v$ we approximate $L_{y}$ by $b v$ and require $\dot{\theta} L_{y}$ to be much smaller than the potential term $4 Q / R$ (which is the dominating one for $r \ll R / 2$ ). If we consider the saddle-point electrons (with momentum $k=v / 2$ ) to originate from, e.g., K-shell ionisation, the relevant impact parameters are of the order of $b \sim 4 k /\left(Q^{2}+k^{2}\right)$ which is the inverse minimum momentum transferred to the electron. The condition $4 Q / R \gg b v^{2} / R$ $\left(>b^{2} v^{2} / R^{2}\right.$ ) implies $Q \geqslant 2 v$, such that the omission of the Coriolis term is justified in the adiabatic to intermediate collision energy regime for impact parameters up to the inverse momentum transfer for inner-shell ionisation.

Note also that, if the wavefunction in the saddle region is known at some time $t_{0}$ such that $\theta(\infty)-\theta\left(t_{0}\right) \ll \pi$, then subsequent evolution of the wavefunction into the asymptotic region can be computed using the propagator with $\dot{\theta} \hat{L}_{y}$ omitted. This is possible even when the Coriolis term may be important for $t<t_{0}$. The discussion in Meckbach et al (1986) employed just this idea, i.e. the wavefunction was assumed known at $t_{0}=R_{0} / v$ with $R_{0} \approx 2 \mathrm{au}$, and was propagated to infinite times using (2.2) with the Coriolis term omitted.

With this approximation, and with the choice of cartesian coordinates for (2.2), the three oscillators decouple. Let $\psi(r, t)=\psi_{1}(x, t) \psi_{2}(y, t) \psi_{3}(z, t)$. Then each of the functions $\psi_{i}$ obeys a differential equation of the type

$$
\begin{aligned}
& \left(\mathrm{i} \frac{\partial}{\partial t}+\frac{1}{2} \frac{\partial^{2}}{\partial x_{i}^{2}}+b_{i}(t) x_{i}^{2}+F_{i}(t)\right) \psi_{i}\left(x_{i}, t\right) \quad i=1,2,3 \\
& b_{1,2}(t)=-8 Q / R^{3} \quad b_{3}(t)=16 Q / R^{3} \quad F_{1}(t)=4 Q / R .
\end{aligned}
$$

The two other functions, $F_{2}$ and $F_{3}$, may be put equal to zero. Apart from a phase factor (due to $F_{1}$ ) the two wavefunctions $\psi_{1}$ and $\psi_{2}$ are solutions to an identical (attractive) oscillator equation, while $\psi_{3}$ belongs to a repulsive oscillator potential of double strength.

\subsection{Harmonic (attractive) oscillator}

In order to calculate the wavefunction $\psi_{1}$ we reduce (2.3) to the Schrödinger equation for a time-independent harmonic oscillator by means of a coordinate transformation, a similar method as has been used in the classical calculations (Nassar et al 1986). To this aim we make the ansatz

$$
\psi_{1}(x, t)=\exp \left(-S(t) x^{2}-Q_{1}(t)\right) u(A(t) x)
$$

which is a generalisation of the conventional form (Peterkop 1971, Feagin 1984, Meckbach et al 1986). Upon substitution into (2.3) for $i=1$ one obtains with $\xi=A(t) x$ the differential equation for the Hermite polynomials

$$
2 n u(\xi)-2 \xi \frac{\mathrm{d} u}{\mathrm{~d} \xi}+\frac{\mathrm{d}^{2} u}{\mathrm{~d} \xi^{2}}=0 \quad n=0,1,2, \ldots
$$

with solution $u_{n}(\xi)=N_{n} H_{n}(\xi), N_{n}$ being a constant (Abramowitz and Stegun 1965, $\mathrm{p}$ 781), provided the functions $S(t), Q_{1}(t)$ and $A(t)$ obey the following relations:

$$
\begin{aligned}
& -\mathrm{i} \dot{S}+2 S^{2}+b_{1}=0 \\
& -\mathrm{i} \dot{Q}_{1}-S+F_{1}=n A^{2} \\
& \mathrm{i} \dot{A}-2 S A=-A^{3}
\end{aligned}
$$


where a dot denotes the time derivative. These equations can be solved in terms of an auxiliary function $w(t)$ which is a solution to the oscillator equation

$$
\ddot{w}-2 b_{1} w=0 \text {. }
$$

Explicitly, one finds

$$
\begin{aligned}
& S=-\frac{\mathrm{i}}{2} \frac{\dot{w}}{w} \quad A^{2}=S+S^{*}=2 \operatorname{Re} S \\
& Q_{1}=\frac{1}{2} \ln \frac{w(t)}{w\left(t_{0}\right)}-\mathrm{i} \int_{t_{0}}^{t} \mathrm{~d} t F_{1}+\frac{1}{2} n \ln \frac{w(t) w^{*}\left(t_{0}\right)}{w\left(t_{0}\right) w^{*}(t)}
\end{aligned}
$$

where $t_{0}$ is some initial time and the integration constant for $Q_{1}$ has been dropped. Thus, the general solution to (2.3) for $i=1$ is given by

$$
\begin{gathered}
\psi_{1 n}(x, t)=N_{n} \exp \left(-S x^{2}\right)\left(\frac{w\left(t_{0}\right)}{w(t)}\right)^{1 / 2} \exp (-\mathrm{in} \varphi) \exp \left(\mathrm{i} \int_{t_{0}}^{t} F_{1} \mathrm{~d} t\right) H_{n}(A x) \\
\varphi(t)=\arg \left(w(t) w^{*}\left(t_{0}\right)\right) \quad N_{n}=\frac{A^{1 / 2}\left(t_{0}\right)}{\pi^{1 / 4}\left(2^{n} n !\right)^{1 / 2}}
\end{gathered}
$$

The constant $N_{n}$ has been determined from the normalisation of $\psi_{1 n}\left(x, t=t_{0}\right)$ to unity.

Like the wavefunction, the propagator defining arbitrary solutions to the Schrödinger equation (2.2) can be factorised in cartesian coordinates

$K\left(\boldsymbol{r}, t ; \boldsymbol{r}_{0}, t_{0}\right)=K_{1}\left(x, t ; x_{0}, t_{0}\right) K_{2}\left(y, t ; y_{0}, t_{0}\right) K_{3}\left(z, t ; z_{0}, t_{0}\right)$

and the propagators $K_{i}$ are constructed from

$$
K_{i}\left(x_{i}, t ; x_{i 0}, t_{0}\right)=\sum_{n} \psi_{i n}\left(x_{i}, t\right) \psi_{i n}^{*}\left(x_{i 0}, t_{0}\right) \quad i=1,2
$$

where the summation runs over the complete set of eigenstates of (2.3). Upon insertion of the solutions (2.9) and with the help of the completeness relation of the eigenfunctions of the stationary harmonic oscillator (Feynman and Hibbs 1965), the propagator in the $x$ direction becomes

$$
\begin{aligned}
K_{1}\left(x, t ; x_{0}, t_{0}\right) & =\frac{A\left(t_{0}\right)}{(2 \pi \mathrm{i} \sin \varphi(t))^{1 / 2}} \exp (\mathrm{i} \varphi(t) / 2)\left(\frac{w\left(t_{0}\right)}{w(t)}\right)^{1 / 2} \exp \left(\mathrm{i} \int_{t_{0}}^{t} F_{1} \mathrm{~d} t\right) \\
& \times \exp \left(-\frac{1}{2}\left[S(t)-S^{*}(t)\right] x^{2}+\frac{1}{2}\left[S\left(t_{0}\right)-S^{*}\left(t_{0}\right)\right] x_{0}^{2}\right) \\
& \times \exp \left(\frac{\mathrm{i}}{2 \sin \varphi(t)}\left[\left(A^{2}(t) x^{2}+A^{2}\left(t_{0}\right) x_{0}^{2}\right) \cos \varphi(t)-2 A\left(t_{0}\right) A(t) x x_{0}\right]\right) .
\end{aligned}
$$

It can be shown that (2.12), together with (2.6) and (2.7), agrees with the result found previously (Khandekar and Lawande 1975, Lawande and Dhara 1983), and that it reduces to the well known result for constant frequency $-2 b_{1}(t) \equiv \omega_{0}^{2}$ (Feynman and Hibbs 1965). The propagator $K_{2}$ is also given by (2.12) with omission of the phase $\exp \left(\mathrm{i} \int_{t_{0}}^{t} F_{1} \mathrm{~d} t\right)$.

Although one may get the impression from expression (2.12) that the propagator depends on the boundary conditions imposed on the system at the point $\left(x_{0}, t_{0}\right)$, this is actually not the case. In order to demonstrate this we rewrite $K_{1}$ by inserting the 
definitions (2.8) for $S$ and $A$ in terms of $w$, and by using the identity $A\left(t_{0}\right) \exp (\mathrm{i} \varphi(t) / 2)\left(w\left(t_{0}\right) / w(t)\right)^{1 / 2}=\left(A(t) A\left(t_{0}\right)\right)^{1 / 2}$ we have

$$
\begin{aligned}
K_{1}\left(x, t ; x_{0}, t_{0}\right) & =\frac{1}{(2 \pi \mathrm{i})^{1 / 2}}\left(\frac{P_{1}\left(t_{0}, t_{0}\right)}{T_{1}\left(t, t_{0}\right)}\right)^{1 / 2} \exp \left(\mathrm{i} \int_{t_{0}}^{t} F_{1} \mathrm{~d} t\right) \\
& \times \exp \left(\frac{\mathrm{i}}{2 T_{1}\left(t, t_{0}\right)}\left[P_{1}\left(t, t_{0}\right) x^{2}+P_{1}\left(t_{0}, t\right) x_{0}^{2}-2 P_{1}\left(t_{0}, t_{0}\right) x x_{0}\right]\right)
\end{aligned}
$$

where these abbreviations have been introduced:

$$
\begin{aligned}
& P_{1}\left(t_{1}, t_{2}\right)=\dot{w}\left(t_{1}\right) w^{*}\left(t_{2}\right)-\dot{w}^{*}\left(t_{1}\right) w\left(t_{2}\right) \\
& T_{1}\left(t_{1}, t_{2}\right)=w\left(t_{1}\right) w^{*}\left(t_{2}\right)-w^{*}\left(t_{1}\right) w\left(t_{2}\right) .
\end{aligned}
$$

The general solution $w(t)$ of the second-order differential equation (2.7) is obtained as a superposition of two linearly independent solutions $w_{a}(t)$ and $w_{b}(t)$ :

$$
w(t)=\lambda_{a} w_{a}(t)+\lambda_{b} w_{b}(t)
$$

with complex constants $\lambda_{a}$ and $\lambda_{b}$. It is immediately seen from the structure of $(2.13)$ that only ratios of the functions $w$ or their derivatives enter into $K_{1}$. Thus, $K_{1}$ will, at most, depend on the ratio $\lambda_{b} / \lambda_{a}$ and we may set $\lambda_{a}=1$. Upon insertion of (2.15) into (2.13) with (2.14), and using real basis functions $w_{a}$ and $w_{b}$, the propagator retains the form (2.13), but now with the definitions

$$
\begin{aligned}
& P_{1}\left(t_{1}, t_{2}\right)=\dot{w}_{a}\left(t_{1}\right) w_{b}\left(t_{2}\right)-\dot{w}_{b}\left(t_{1}\right) w_{a}\left(t_{2}\right) \\
& T_{1}\left(t_{1}, t_{2}\right)=w_{a}\left(t_{1}\right) w_{b}\left(t_{2}\right)-w_{b}\left(t_{1}\right) w_{a}\left(t_{2}\right) .
\end{aligned}
$$

The complete arbitrariness of the functions $w_{a}$ and $w_{b}$ (apart from the required linear independence) ensures that the propagator does not depend on the boundary conditions.

\subsection{Inverse harmonic oscillator}

We now proceed to the solutions of (2.3) for $i=3$, i.e. with a positive potential. Insertion of the ansatz

$$
\psi_{3}(z, t)=\exp \left(-\mathrm{i} S_{3}(t) z^{2}-Q_{3}(t)\right) u_{3}\left(A_{3}(t) z\right)
$$

with real functions $S_{3}$ and $A_{3}$ into (2.3) leads to the following differential equation:

$$
\frac{\mathrm{d}^{2} u_{3}}{\mathrm{~d} \zeta^{2}}-2 \mathrm{i} \zeta \frac{\mathrm{d} u_{3}}{\mathrm{~d} \zeta}+2 \kappa u_{3}(\zeta)=0 \quad \zeta=A_{3}(t) z
$$

Its solutions $u_{3}(\zeta)=N_{\varepsilon} \exp \left(\mathrm{i} \zeta^{2} / 2\right) E^{*}(-\varepsilon, \sqrt{2} \zeta)$ can be expressed in terms of the parabolic cylinder functions $E^{*}$ to positive energy $\varepsilon$, where $\kappa=\varepsilon-\mathrm{i} / 2$, and $N_{\varepsilon}$ a constant (Barton 1986, Abramowitz and Stegun 1965, p 692). Equation (2.18) holds, provided the functions $S_{3}, Q_{3}$ and $A_{3}$ obey the relations

$$
\begin{aligned}
& \dot{S}_{3}-2 S_{3}^{2}+b_{3}=0 \\
& \mathrm{i} \dot{A}_{3}-2 \mathrm{i} S_{3} A_{3}=-\mathrm{i} A_{3}^{3} \\
& -\mathrm{i} \dot{Q}_{3}-\mathrm{i} S_{3}=\kappa A_{3}^{2} .
\end{aligned}
$$

Let $v_{a}$ and $v_{b}$ be two linearly independent real solutions of the auxiliary equation

$$
\ddot{v}-2 b_{3} v=0 \text {. }
$$


Then the solutions to (2.19) are obtained similarly as in $\$ 2$ in the following form:

$$
\begin{aligned}
& S_{3}=-\frac{1}{2} v_{a} / v_{a} \quad A_{3}^{2}=S_{3}+\hat{S}_{3} \quad \hat{S}_{3}=\frac{1}{2} \dot{v}_{b} / v_{b} \\
& Q_{3}=\frac{1}{2} \ln \frac{v_{a}(t)}{v_{a}\left(t_{0}\right)}-\left(\mathrm{i} \varepsilon+\frac{1}{2}\right) \frac{1}{2} \ln \frac{v_{a}(t) v_{b}\left(t_{0}\right)}{v_{a}\left(t_{0}\right) v_{b}(t)}
\end{aligned}
$$

with $t_{0}$ defining some initial time. From this, the general solution to the inverse harmonic oscillator is obtained:

$$
\begin{aligned}
& \psi_{\varepsilon}(z, t)=N_{\varepsilon} \exp \left(-\mathrm{i} S_{3} z^{2}\right)\left(\frac{v_{a}\left(t_{0}\right)}{v_{a}(t)}\right)^{1 / 2} \phi(t)^{-\frac{1}{2} i \varepsilon-\frac{1}{4}} \exp \left(\frac{1}{2} \mathrm{i} A_{3}^{2} z^{2}\right) E^{*}\left(-\varepsilon, \sqrt{2} A_{3} z\right) \\
& \phi(t)=\frac{v_{a}\left(t_{0}\right) v_{b}(t)}{v_{a}(t) v_{b}\left(t_{0}\right)} \quad N_{\varepsilon}=\frac{2^{1 / 4} A_{3}^{1 / 2}\left(t_{0}\right)}{(4 \pi)^{1 / 2}[1+\exp (-2 \pi \varepsilon)]^{1 / 2}}
\end{aligned}
$$

The normalisation constant $N_{\varepsilon}$ has been chosen such that $\psi_{\varepsilon}$ is $\delta$-normalised in energy at $t=t_{0}$ (Barton 1986).

Generalising the results of the time-independent inverse oscillator (Barton 1986), the propagator $K_{3}$ is readily obtained:

$$
\begin{aligned}
K_{3}\left(z, t ; z_{0}, t_{0}\right)= & \int_{-\infty}^{\infty} \mathrm{d} \varepsilon \psi_{\varepsilon}(z, t) \psi_{\varepsilon}^{*}\left(z_{0}, t_{0}\right) \\
= & \frac{A_{3}\left(t_{0}\right)}{\left[2 \pi \mathrm{i} \sinh \left(\frac{1}{2} \ln \phi(t)\right)\right]^{1 / 2}} \phi(t)^{-1 / 4}\left(\frac{v_{a}\left(t_{0}\right)}{v_{a}(t)}\right)^{1 / 2} \\
& \times \exp \left(-\frac{1}{2} \mathrm{i}\left[S_{3}(t)-\hat{S}_{3}(t)\right] z^{2}+\frac{1}{2} \mathrm{i}\left[S_{3}\left(t_{0}\right)-\hat{S}_{3}\left(t_{0}\right)\right] z_{0}^{2}\right) \\
& \times \exp \left(\frac { \mathrm { i } } { 2 \operatorname { s i n h } ( \frac { 1 } { 2 } \operatorname { l n } \phi ( t ) ) } \left[\left(A_{3}^{2}(t) z^{2}+A_{3}^{2}\left(t_{0}\right) z_{0}^{2}\right) \cosh \left(\frac{1}{2} \ln \phi(t)\right)\right.\right. \\
& \left.\left.-2 A_{3}\left(t_{0}\right) A_{3}(t) z z_{0}\right]\right) .
\end{aligned}
$$

This expression is basically of the same structure as the propagator (2.12) for the attractive oscillator. In fact, since $A_{3}\left(t_{0}\right)\left(v_{a}\left(t_{0}\right) / v_{a}(t)\right)^{1 / 2} \phi(t)^{-1 / 4}=\left(A_{3}(t) A_{3}\left(t_{0}\right)\right)^{1 / 2}$, $K_{3}$ can be cast into a form identical to $(2.13)$ :

$$
\begin{aligned}
& K_{3}\left(z, t ; z_{0}, t_{0}\right)=\frac{1}{(2 \pi \mathrm{i})^{1 / 2}}\left(\frac{P_{3}\left(t_{0}, t_{0}\right)}{T_{3}\left(t, t_{0}\right)}\right)^{1 / 2} \\
& \times \exp \left(\frac{\mathrm{i}}{2 T_{3}\left(t, t_{0}\right)}\left[P_{3}\left(t, t_{0}\right) z^{2}+P_{3}\left(t_{0}, t\right) z_{0}^{2}-2 P_{3}\left(t_{0}, t_{0}\right) z z_{0}\right]\right) \\
& P_{3}\left(t_{1}, t_{2}\right)=\dot{v}_{a}\left(t_{1}\right) v_{b}\left(t_{2}\right)-\dot{v}_{b}\left(t_{1}\right) v_{a}\left(t_{2}\right) \quad T_{3}\left(t_{1}, t_{2}\right)=v_{a}\left(t_{1}\right) v_{b}\left(t_{2}\right)-v_{b}\left(t_{1}\right) v_{a}\left(t_{2}\right) .
\end{aligned}
$$

The sign of the quadratic potential is thus irrelevant for the construction of the propagator; it enters only into the differential equation for the auxiliary functions (2.7) or $(2.20)$.

\section{Solutions of the oscillator equation for specific time dependencies}

The explicit time dependence of the propagator is obtained by solving the auxiliary differential equation:

$$
\frac{\mathrm{d}^{2} w}{\mathrm{~d} t^{2}}+\frac{16 Q \lambda_{\mathrm{i}}}{R(t)^{3}} w=0 \quad \lambda_{i}=\left\{\begin{array}{rl}
1 & i=1,2 \\
-2 & i=3 .
\end{array}\right.
$$


Recalling that in the underlying physical problem, $R(t)$ describes the internuclear distance, we shall solve (3.1) for four cases: $(a)$ straight-line trajectory with zero impact parameter, $(b)$ straight-line trajectory with non-zero impact parameter, $(c)$ Coulomb trajectory with zero impact parameter, and $(d)$ Coulomb trajectory with $b \neq 0$.

\subsection{Straight-line trajectory with $b=0: R=v|t|$}

We include this simplest case of time dependence only for the sake of completeness, as it is closely related to the Wannier theory of threshold ionisation (Peterkop 1971, Feagin 1984) and has been solved previously (Meckbach et al 1986).

Making in (3.1) the substitution $x=1 / t$ and introducing $\beta=16 Q \lambda_{i} / v^{3}$ one obtains

$$
x^{2} \frac{\mathrm{d}^{2} w}{\mathrm{~d} x^{2}}+2 x \frac{\mathrm{d} w}{\mathrm{~d} x}+\beta x w(x)=0
$$

Its solution can be expressed in terms of the Bessel functions $w(x)=$ $|x|^{-1 / 2} Z_{1}\left(2(\beta|x|)^{1 / 2}\right)$ (Kamke 1967, p 440). Thus two linearly independent solutions are

$$
w_{a}(t)=|t|^{1 / 2} J_{1}\left(2(\beta /|t|)^{1 / 2}\right) \quad w_{b}(t)=|t|^{1 / 2} Y_{1}\left(2(\beta /|t|)^{1 / 2}\right)
$$

$J_{1}$ and $Y_{1}$ being Bessel functions of the first and second kind, respectively (Abramowitz and Stegun 1965, p 358).

\subsection{Straight-line trajectory with $b \neq 0: R=\left(b^{2}+v^{2} t^{2}\right)^{1 / 2}$}

We introduce a new variable $y=1-b / R$ with the property $y=0$ at $t=0$ and obtain from (3.1)

$$
\left(y^{3}-3 y^{2}+2 y\right) \frac{\mathrm{d}^{2} w}{\mathrm{~d} y^{2}}+\left(3 y^{2}-6 y+1\right) \frac{\mathrm{d} w}{\mathrm{~d} y}+\hat{\alpha} w(y)=0
$$

with $\hat{\alpha}=16 Q \lambda_{i} /\left(v^{2} b\right)$. This is a special case of Heun's differential equation, which in its general form is (Kamke 1967, p 485)

$$
\begin{aligned}
y(y-1)(y-a) & \frac{\mathrm{d}^{2} h}{\mathrm{~d} y^{2}}+\left\{(\alpha+\beta+1) y^{2}-[\alpha+\beta+1+a(\gamma+\delta)-\delta] y+a \gamma\right\} \frac{\mathrm{d} h}{\mathrm{~d} y} \\
& +(\alpha \beta y-q) h(y)=0 .
\end{aligned}
$$

Its solution, the function $h(a, q ; \alpha, \beta, \gamma, \delta, y)$ depends on the parameters introduced in (3.5). This function resembles in many aspects the hypergeometric function (Kamke 1967).

For arguments $|y|<1$ the first solution of (3.5), which will be termed the Heun function, is defined by the series representation:

$$
\begin{aligned}
& h_{1}(y) \equiv h(a, q ; \alpha, \beta, \gamma, \delta, y)=1+\sum_{n=1}^{\infty} c_{n} y^{n} \quad c_{0}=1 \quad c_{1}=q /(a \gamma) \\
& a(n+1)(\gamma+n) c_{n+1} \\
& =[a(\gamma+\delta+n-1)+\alpha+\beta-\delta+n+q / n] n c_{n} \\
& -[(n-1)(n-2)+(n-1)(\alpha+\beta+1)+\alpha \beta] c_{n-1} \quad n \geqslant 1
\end{aligned}
$$


if $|a| \geqslant 1$ and $\gamma \neq 0,-1,-2, \ldots$ The second solution of $(3.5)$, which is linearly independent of (3.6), is for $|\gamma| \neq 0,1,2, \ldots$, given by

$$
\begin{aligned}
& h_{2}(y)=|y|^{1-\gamma} h\left(a, q_{1} ; \alpha-\gamma+1, \beta-\gamma+1,2-\gamma, \delta, y\right) \\
& q_{1}=q+(\alpha-\gamma+1)(\beta-\gamma+1)-\alpha \beta+\delta(\gamma-1)(1-a) .
\end{aligned}
$$

If the argument $|y| \geqslant 1$ or $|a|<1$, the solution to (3.5) is obtained from transformation formulas of the Heun function (Kamke 1967, p 486).

For the special case of our differential equation (3.4), the two solutions for $y<1$ are given by

$$
\begin{aligned}
& w_{a}(y)=\sum_{n=0}^{\infty} c_{n} y^{n} \quad c_{0}=1 \quad c_{1}=-\hat{\alpha} \\
& c_{n}=\frac{1}{n(2 n-1)}\left\{[3 n(n-1)-\hat{\alpha}] c_{n-1}-n(n-2) c_{n-2}\right\} \quad n \geqslant 2 \\
& w_{b}(y)=\left\{\left.y\right|^{1 / 2} \sum_{n=0}^{\infty} g_{n} y^{n} \quad g_{0}=1 \quad g_{1}=\frac{1}{3}\left(\frac{9}{4}-\hat{\alpha}\right)\right. \\
& g_{n}=\frac{1}{n(2 n+1)}\left\{\left[3\left(n^{2}-\frac{1}{4}\right)-\hat{\alpha}\right] g_{n-1}-\left(n-\frac{3}{2}\right)\left(n+\frac{1}{2}\right) g_{n-2}\right\} \quad n \geqslant 2 .
\end{aligned}
$$

For $|t| \rightarrow \infty$ one has $y \rightarrow 1$, such that the transformation formulae have to be used. Equivalently, a variable transformation $x=1-y=b / R$ can be applied to (3.4), such that $x \rightarrow 0$ for $|t| \rightarrow \infty$. The solutions valid for $x<1$ are

$$
\begin{aligned}
& \hat{w}_{a}(x)=1+\sum_{n=1}^{\infty} d_{n} x^{n} \quad d_{0}=1 \quad d_{1}=-\frac{1}{2} \hat{\alpha} \quad n \geqslant 2 \quad d_{-1}=0 \\
& d_{n}=\frac{1}{n(n+1)}\left[n(n-2) d_{n-2}-\hat{\alpha} d_{n-1}\right] \quad b_{0}=-1 / \hat{\alpha} \quad b_{1}=0 \\
& \hat{w}_{b}(x)=\hat{w}_{a}(x) \ln |x|+b_{0} / x+\sum_{n=2}^{\infty} b_{n} x^{n-1} \quad n \geqslant 2 . \\
& b_{n}=\frac{1}{n(n-1)}\left[2(n-2) d_{n-3}-(2 n-1) d_{n-1}+(n-3)(n-1) b_{n-2}-\hat{\alpha} b_{n-1}\right] \quad n \geqslant 2
\end{aligned}
$$

As the solution $\hat{w}_{a}$ corresponds to an integer parameter $\gamma$, the function $\hat{w}_{b}$ had to be found as in the case of the hypergeometric differential equation from, for example, the method of Frobenius (Kamke 1967, p 82).

In order to extend the solutions $w_{a}(y)$ and $w_{b}(y)$ to $y \rightarrow 1$, they have to be expanded in the basis $\left\{\hat{w}_{a}(1-y), \hat{w}_{b}(1-y)\right\}$, the expansion coefficients being obtained from the matching condition at two intermediate points (e.g. at $y=0.4,0.5$ ). Figure 1 illustrates the behaviour of $w_{a}(y)$ for different values of the parameter $\hat{\alpha}$. $w_{a}(y)=1$ for $y=0$ and diverges at $y=1$ like $(y-1)^{-1}$ in addition to a logarithmic singularity (cf (3.9)). The second solution $w_{b}(y)$ behaves qualitatively similar to $w_{a}(y)$, except that it is zero (and non-analytical) at $y=0$. Increasing the modulus of the parameter $\hat{\alpha}$ introduces more and more oscillations, together with an increase of the functional values at the extrema.

It should be noted that the $b=0$ case cannot trivially be obtained as a special case of the above solutions, since $b$ enters as a scale parameter into (3.4). 


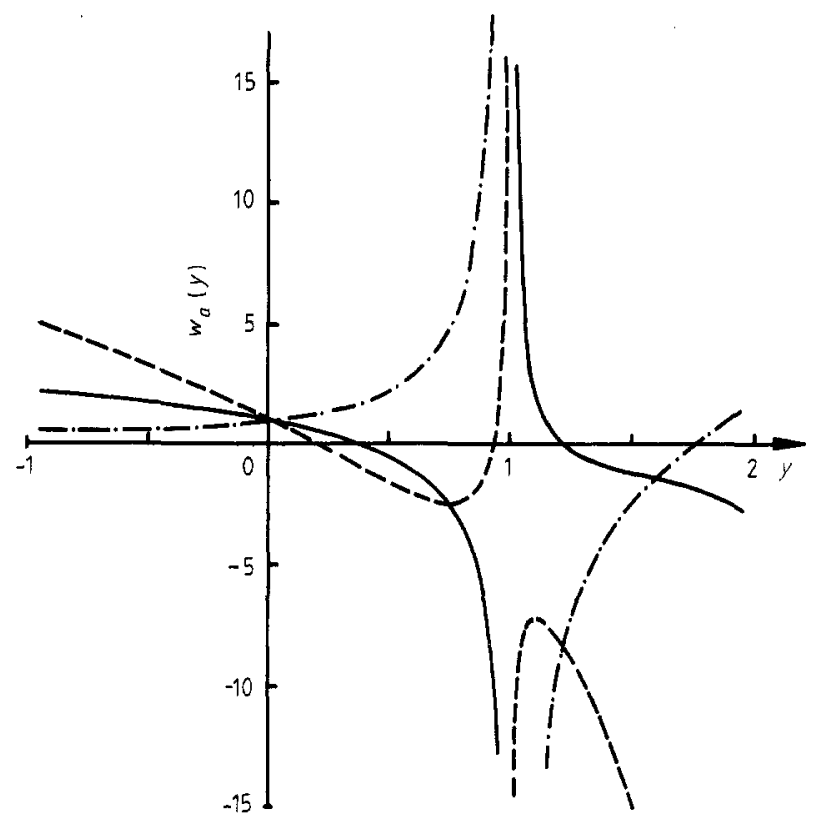

Figure 1. The Heun function $w_{a}(y)=h\left(2,-\hat{\alpha} ; 0,2, \frac{1}{2}, 2, y\right)$ for different values of the parameter $\hat{\alpha} .-\cdots: \hat{\alpha}=-1 ;-: \hat{\alpha}=2 ;-\cdots ; \hat{\alpha}=5$. In the present context, the variable $y$ is restricted to $0 \leqslant y<1$.

3.3. Coulomb trajectory with $b=0: R=d(1+\varepsilon \cosh \tau), t=(d / v)(\varepsilon \sinh \tau+\tau)$

The Coulomb trajectory in its parametric representation $(-\infty<\tau<\infty)$ is characterised by the internuclear distance $2 d$ in a head-on collision and by the eccentricity $\varepsilon$ which is unity for $b=0$.

Introducing the new variable $\rho=R / 2 d=\frac{1}{2}(1+\varepsilon \cosh \tau)$, the differential equation (3.1) transforms into

$\left[\rho^{3}-\rho^{2}+\frac{1}{4} \rho\left(1-\varepsilon^{2}\right)\right] \frac{\mathrm{d}^{2} w}{\mathrm{~d} \rho^{2}}+\left[\frac{1}{2} \rho-\frac{1}{4}\left(1-\varepsilon^{2}\right)\right] \frac{\mathrm{d} w}{\mathrm{~d} \rho}+\frac{8 Q \lambda_{i}}{\mathrm{~d} v^{2}} w(\rho)=0$.

For zero impact parameter, i.e. $\varepsilon=1$, this equation reduces to

$$
\rho^{2}(\rho-1) \frac{\mathrm{d}^{2} w}{\mathrm{~d} \rho^{2}}+\frac{\rho}{2} \frac{\mathrm{d} w}{\mathrm{~d} \rho}+\frac{8 Q \lambda_{i}}{\mathrm{~d} v^{2}} w(\rho)=0 .
$$

This equation is also of Heun type (3.5), but as we have the special case of the parameter $a=0$, a further reduction of the Heun function to the hypergeometric function ${ }_{2} F_{1}$ is possible (Kamke 1967, p 470). The two solutions are

$$
\begin{aligned}
& w_{a}(\rho)=|\rho|_{2}^{\alpha} F_{1}(\alpha, \alpha-1, \alpha-\beta+1, \rho) \\
& w_{b}(\rho)=|\rho|_{2}^{\beta} F_{1}(\beta, \beta-1, \beta-\alpha+1, \rho) \\
& \alpha, \beta=\frac{3}{4} \pm \frac{1}{2}\left(\frac{9}{4}+32 Q \lambda_{1} / \mathrm{d} v^{2}\right)^{1 / 2} \quad \rho<1 .
\end{aligned}
$$

Since in our case $\alpha \neq \beta$, the two functions $w_{a}$ and $w_{b}$ are indeed linearly independent. For $\rho \geqslant 1$, the appropriate transformation formulae have to be used (Abramowitz and Stegun 1965, p 559). 


\subsection{Coulomb trajectory with $b \neq 0$}

In this case we have to solve (3.10) for $\varepsilon>1$. This equation is related to Heun's equation, as can be seen through a variable transformation $\xi=(1+\varepsilon) / 2 \varepsilon-\rho / \varepsilon=$ $\frac{1}{2}(1-\cosh \tau)($ Kamke 1967, p 487). The solution is

$$
\begin{aligned}
& w_{a}(\xi)=h\left(\frac{2 \varepsilon}{1+\varepsilon}, \frac{16 Q \lambda_{i}}{\mathrm{~d} v^{2}(1+\varepsilon)} ; 0,-1, \frac{1}{2},-1, \frac{2 \varepsilon}{1+\varepsilon} \xi\right) \\
& w_{b}(\xi)=|\xi|^{1 / 2} h\left(\frac{2 \varepsilon}{1+\varepsilon}, q_{1} ; \frac{1}{2},-\frac{1}{2}, \frac{3}{2},-1, \frac{2 \varepsilon}{1+\varepsilon} \xi\right) \\
& q_{1}=\frac{16 Q \lambda_{i}}{\mathrm{~d} v^{2}(1+\varepsilon)}-\frac{1}{4}+\frac{1-\varepsilon}{2(1+\varepsilon)} .
\end{aligned}
$$

For $|2 \varepsilon /(1+\varepsilon) \xi| \geqslant 1$, transformation formulae, as well as the method of Frobenius, have to be applied for the construction of the solutions.

The special case of $b=0$ (i.e. $\varepsilon=1$ ) is easily recovered by using, instead of $w_{a}(\xi)$, the transformed solution

$$
\hat{w}_{a}(\xi)=h\left(0,-\frac{8 Q \lambda_{i}}{\mathrm{~d} v^{2}} ; 0,-1,-1, \frac{1}{2}, \rho\right) \quad \rho=1-\xi
$$

which can be expressed as a linear combination of the solutions $w_{a}(\rho)$ and $w_{b}(\rho)$ from (3.12).

\section{Calculation of the electronic wavefunction}

The wavefunction $\psi(\boldsymbol{r}, t)$ can be represented by a linear combination of solutions to the Schrödinger equation (2.2), with expansion coefficients $a_{\kappa}$ which are determined from the initial condition $\psi(\boldsymbol{r}, t)=\psi\left(\boldsymbol{r}, t_{0}\right)$ at time $t_{0}$. Thus it can be expressed in terms of the propagator

$$
\psi(\boldsymbol{r}, t)=\oint_{\kappa} a_{\kappa} \psi_{\kappa}(\boldsymbol{r}, t)=\int \mathrm{d} \boldsymbol{r}_{0} K\left(\boldsymbol{r}, t ; \boldsymbol{r}_{0}, t_{0}\right) \psi\left(\boldsymbol{r}_{0}, t_{0}\right)
$$

where the definitions from (2.10), (2.11) and (2.23) have been used, and $\kappa$ comprises the quantum numbers $n$ and energies $\varepsilon$. Due to the Gaussian-type $\boldsymbol{r}_{0}$ dependence of $K$, this integral is easily evaluated. As a first example, we consider a wavefunction which develops from a plane wave

$$
\psi_{k}\left(\boldsymbol{r}_{0}, t_{0}\right)=\frac{1}{(2 \pi)^{3 / 2}} \exp \left(\mathrm{i} \boldsymbol{k} \cdot \boldsymbol{r}_{0}\right) \exp \left(-\mathrm{i} k^{2} t_{0} / 2\right)
$$

which is a solution to the Schrödinger equation at $t_{0} \rightarrow-\infty$ (apart from a Coulomb phase). With this, the wavefunction is calculated from (4.1) to be

$$
\begin{aligned}
\psi_{k}(\boldsymbol{r}, t)=\frac{1}{(2 \pi)^{3 / 2}} & \exp \left(\mathrm{i} \int_{t_{0}}^{t} \frac{4 Q}{R} \mathrm{~d} t\right) \exp \left(-\mathrm{i} k^{2} t_{0} / 2\right) \prod_{j=1}^{3}\left(\frac{P_{j}\left(t_{0}, t_{0}\right)}{P_{j}\left(t_{0}, t\right)}\right)^{1 / 2} \\
& \quad \times \exp \left(\frac{\mathrm{i}}{2 T_{j}\left(t, t_{0}\right)} P_{j}\left(t, t_{0}\right) x_{j}^{2}\right) \exp \left[-\mathrm{i} \frac{T_{j}\left(t, t_{0}\right)}{2 P_{j}\left(t_{0}, t\right)}\left(k_{j}-\frac{P_{j}\left(t_{0}, t_{0}\right)}{T_{j}\left(t, t_{0}\right)} x_{j}\right)^{2}\right]
\end{aligned}
$$


with $P_{2}=P_{1}$ and $T_{2}=T_{1}$ from (2.16). If one employs, for example, the time dependence of a $(b \neq 0)$ straight-line path, it is found that, for small times $t \simeq 0$, the wave travels with a momentum different from $k$, apart from a phase depending quadratically on the coordinates. At $t \rightarrow+\infty, \psi_{k}(r, t)$ returns basically into the plane-wave form (4.2), having accumulated a Coulomb phase.

As a second example we consider the motion of a Gaussian wavepacket, which has been investigated previously for a $b=0$ straight-line path (Meckbach et al 1986):

$$
\psi_{\beta}\left(\boldsymbol{r}_{0}, t_{0}\right)=(\beta / \sqrt{\pi})^{3 / 2} \exp \left(-\frac{1}{2} \beta^{2} r_{0}^{2}\right) \exp \left(-3 \mathrm{i} \beta^{2} t_{0} / 2\right)
$$

From (4.1) we then obtain

$$
\begin{aligned}
\psi_{\beta}(\boldsymbol{r}, t)=\left(\frac{\beta}{\mathrm{i} \sqrt{\pi}}\right)^{3 / 2} & \exp \left(\mathrm{i} \int_{t_{0}}^{t} \frac{4 Q}{R} \mathrm{~d} t\right) \exp \left(-3 \mathrm{i} \beta^{2} t_{0} / 2\right) \prod_{j=1}^{3}\left(\frac{P_{j}\left(t_{0}, t_{0}\right)}{\beta^{2} T_{j}\left(t, t_{0}\right)-\mathrm{i} P_{j}\left(t_{0}, t\right)}\right)^{1 / 2} \\
& \times \exp \left[\frac{x_{j}^{2}}{2 T_{j}\left(t, t_{0}\right)}\left(\mathrm{i} P_{j}\left(t, t_{0}\right)-\frac{P_{j}^{2}\left(t_{0}, t_{0}\right)}{\beta^{2} T_{j}\left(t, t_{0}\right)-i P_{j}\left(t_{0}, t\right)}\right)\right] .
\end{aligned}
$$

Taking, for example, $t_{0}=0$, it is found that, for large times $t$, the width of the wavepacket increases with $t$ while the normalisation factor decreases with $t^{-3 / 2}$, which means that the electronic density distribution eventually spreads over the whole space, albeit differently in $(x, y)$ and $z$ direction (Meckbach et al 1986). This does, however, not influence the norm of the wavefunction, which is unity.

\section{Concluding remarks}

In a reference frame fixed at the saddle point of the two-centre Coulomb potential, we have shown that the Schrödinger equation for an electron can be solved exactly for some classes of time dependencies of the internuclear distance, if the Coulomb field is expanded up to second order around the origin. The functional dependence of the corresponding propagator is found to be independent of the sign of the potential, such that the three spatial coordinates can be treated on the same footing. For an input time dependence of a straight-line motion or a Coulomb trajectory, the relevant function which governs the time dependence of the wavefunction is the Heun function, which in special cases reduces to the hypergeometric function.

In a physical situation of interest, the wavefunction has to be known in the laboratory frame of reference, i.e. the target rest system. For collinear moving nuclei, e.g. for zero impact parameter trajectories, the target-centred wavefunction $\psi_{\mathrm{T}}\left(\boldsymbol{r}_{\mathrm{T}}, t\right)$ is simply obtained from the saddle-point-centred function $\psi(\boldsymbol{r}, t)$ by a frame transformation, since the saddle-point frame coincides with the centre-of-mass frame (which is an inertial system)

$$
\psi_{\mathrm{T}}\left(\boldsymbol{r}_{\mathrm{T}}, t\right)=\exp \left(-\mathrm{i} \int \mathrm{d} t \dot{R}^{2} / 8\right) \exp \left(\mathrm{i} \dot{\boldsymbol{R}}_{\mathrm{T}} / 2\right) \psi\left(\boldsymbol{r}_{\mathrm{T}}-\boldsymbol{R} / 2, t\right)
$$

with $\psi(\boldsymbol{r}, t)$ from (4.1). For a planar internuclear motion $(b \neq 0)$, on the other hand, the saddle-point system has to be considered as a rotating reference frame, since the $z$ axis is taken along the internuclear coordinate $\boldsymbol{R}$. In this respect, the function $\psi(\boldsymbol{r}, t)$ is-in contrast to the $b=0$ case-only an approximate solution, because the centrifugal term $\dot{\theta} \hat{L}_{y}$ in the Schrödinger equation has been omitted. The transformation to the target frame includes a rotation, in addition to the translation (5.1). 


\section{Acknowledgments}

Helpful discussions with P A Amundsen are gratefully acknowledged. This work was supported in part by the Gesellschaft für Schwerionenforschung Darmstadt. One of us (JM) acknowledges support by the National Science Foundation under grant no PHY-860298.

\section{References}

Abramowitz M and Stegun I A 1965 Handbook of Mathematical Functions (New York: Dover)

Barton G 1986 Ann. Phys., NY 166322

Feagin J M 1984 J. Phys. B: At. Mol. Phys. 172433

Feynman R P and Hibbs H R 1965 Quantum Mechanics and Path Integrals (New York: McGraw-Hill) p 200

Kamke E 1967 Differentialgleichungen, Lösungsmethoden und Lösungen (Leipzig: Akademische Verlagsgesellschaft)

Khandekar D C and Lawande S V 1975 J. Math. Phys. 16384

Lawande S V and Dhara A K 1983 Phys. Lett. 99A 353

Meckbach W, Focke P J, Goñi A R, Suárez S, Macek J and Menendez M G 1986 Phys. Rev. Lett. 571587

Nassar A B, Bassalo J M F and de Tarso S Alencar P 1986 Phys. Lett. 113A 365

Nassar A B and Berg R T 1986 Phys. Rev. A 342462

Olson R E, Gay T J, Berry H G, Hale E B and Irby V D 1987 Phys. Rev. Lett. 5936

Peterkop R 1971 J. Phys. B: At. Mol. Phys. 4513

Stolterfoht N, Schneider D, Tanis J, Altevogt H, Salin A, Fainstein P D, Rivarola R, Grandin J P, Scheurer J N, Andriamonje S, Bertault D and Chemin J F 1987 Europhys. Lett. 4899

Suárez S, Meckbach W, Bernardi G C and Focke P 1988 Z. Phys. D 7309 